CRYSTALLOGRAPHIC COMMUNICATIONS

ISSN 2056-9890

Received 27 November 2014

Accepted 22 December 2014

Edited by V. V. Chernyshev, Moscow State University, Russia

Keywords: crystal structure; coumarin derivatives; dibromation; hydrogen bonding; $\pi-\pi$ interactions

CCDC reference: 1040655

Supporting information: this article has supporting information at journals.iucr.org/e

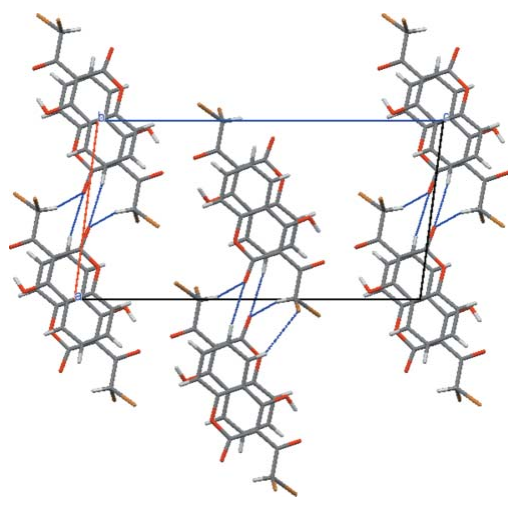

OPEN $\odot$ ACCESS

\section{Crystal structure of 3-(2,2-dibromoacetyl)-4- hydroxy-2H-chromen-2-one}

\author{
Ameni Brahmia, Afef Ghouili and Rached Ben Hassen*
}

Unité de Chimie des Matériaux et de I'Environnement UR11ES25, ISSBAT, Université de Tunis-El Manar, 9, Avenue Dr. Zoheir SAFI, 1006 Tunis, Tunisia. *Correspondence e-mail: rached.benhassen@fss.rnu.tn

The title compound, $\mathrm{C}_{11} \mathrm{H}_{6} \mathrm{Br}_{2} \mathrm{O}_{4}$, is a new coumarin derivative obtained from the reaction of 3-acetyl-4-hydroxy- $2 H$-chromen-2-one with bromine in acetic acid. The hydroxyl group in involved in an intramolecular $\mathrm{O}-\mathrm{H} \cdots \mathrm{O}$ hydrogen bond. In the crystal, $\pi-\pi$ interactions between the rings of the bicycle [intercentroid distances $=3.498$ (2) and 3.539 (2) $\AA$ ] pack molecules into stacks along the $b$ axis, and weak intermolecular $\mathrm{C}-\mathrm{H} \cdots \mathrm{O}$ hydrogen bonds further link these stacks into layers parallel to the $a b$ plane.

\section{Chemical context}

3-Acetyl-4-hydroxy-2H-chromen-2-one is one of the wellknown 3-substituted-4-hydroxycoumarins, which form a class of fused-ring heterocycles and occur widely among natural products. Several natural products with the coumarinic moiety exhibit interesting biological properties such as anti-oxidant and antibacterial (Kayser \& Kolodziej, 1997). They also possess pharmacological activities including anti-inflammatory (Mahidol et al., 2004), anticancer (Wang et al., 2002) and inhibition of platelet aggregation (Cravotto et al., 2001). These derivatives are very susceptible to electrophilic substitutions (Dou et al., 1969); their reaction with bromine can give rise to several compounds used as intermediate products which are susceptible to interesting substitutions (Takase et al., 1971) in a wide range of organic syntheses. The bromination of these compounds increases their anticonvulsant activity (Dimmock et al., 2000), which gives them pharmacological importance. Thus, as part of a study of the effects of substituents on the crystal structures of 3-acetyl-4-hydroxycoumarins (Traven et $a l ., 2000)$, the structure of 3-(2,2-dibromoacetyl)-4-hydroxy$2 \mathrm{H}$-chromen-2-one, (I), has been determined.<smiles>O=C1Oc2ccccc2C(O)C1C(=O)C(Br)Br</smiles>

\section{Structural commentary}

In the title compound (Fig. 1), the hydroxy group is involved in formation of an intramolecular $\mathrm{O}-\mathrm{H} \cdots \mathrm{O}$ hydrogen bond (Table 1). In fact, the O3-H5 distance of 0.94 (7) $\AA$ has decreased from 1.02 (3) $\AA$, observed in the starting reagent 3-acetyl-4-hydroxy-2H-chromen-2-one (Lyssenko \& Antipin, 2001). The H5 ..O4 distance of $1.65(7) \AA$ is elongated 


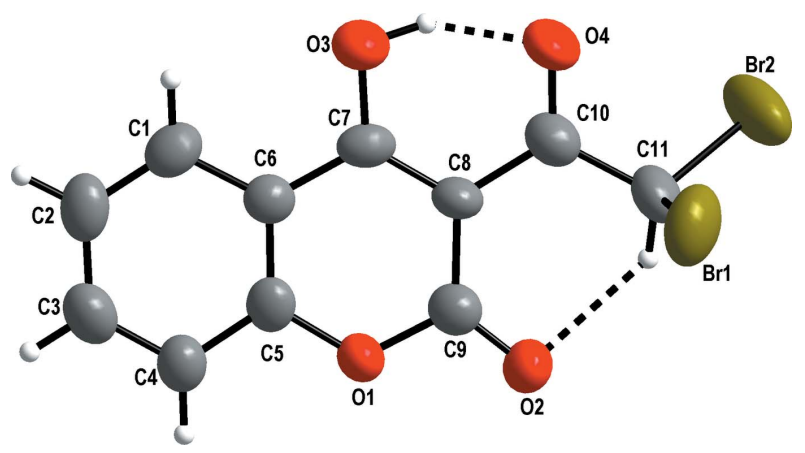

Figure 1

The molecular structure of (I), showing the atom-numbering scheme. Displacement ellipsoids are drawn at the $50 \%$ probability level. Intramolecular hydrogen bonds are shown as dashed lines.

compared with its value in the parent compound [1.45 (3) $\mathrm{A}]$, and the $\mathrm{O} 3-\mathrm{H} 5 \cdots \mathrm{O} 4$ angle of $147(6)^{\circ}$ is significantly smaller than that found for the starting reagent $\left[161(2)^{\circ}\right]$. This trend has already been observed in the fluorinated compound 2-difluoroacetyl-1,3-cyclohexadione (Grieco et al., 2011), in which the $\mathrm{O} 3-\mathrm{H} 5$ and $\mathrm{H} 5 \cdots \mathrm{O} 4$ distances are even more affected ( 0.908 and $1.658 \AA$ respectively). These observations can be easily understood from the point of view of the strong attractive effect of the halogen atoms due to their high electronegativities. All these geometrical parameters are in good agreement with the significant attractor effect of the halogen atoms, which affects the lone pairs of the oxygen atom $\mathrm{O} 4$, leading to a decrease of the attractor effect of $\mathrm{O} 4$ in the $\mathrm{H} 5 \cdots \mathrm{O} 4$ hydrogen bond and, consequently, an increase in the H5 ...O4 distance.

The $\mathrm{C}-\mathrm{C}$ and $\mathrm{C}-\mathrm{O}$ bond lengths in (I) correspond well to those observed in the parent compound, so they are not affected by the $\alpha$-ketodibromation except for $\mathrm{C} 10-\mathrm{C} 11$ [1.523 (9) $\AA$ ] which is elongated compared to the distance in the starting reagent [1.485 (2) A; Lyssenko \& Antipin, 2001).

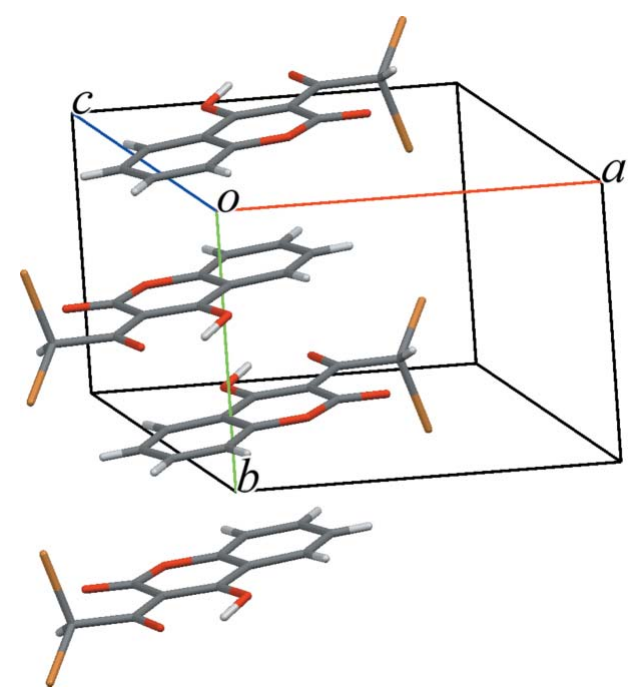

Figure 2

A portion of the crystal packing showing one stack of molecules parallel to the $b$ axis.
Table 1

Hydrogen-bond geometry $\left(\AA{ }^{\circ}\right)$.

\begin{tabular}{lllll}
\hline$D-\mathrm{H} \cdots A$ & $D-\mathrm{H}$ & $\mathrm{H} \cdots A$ & $D \cdots A$ & $D-\mathrm{H} \cdots A$ \\
\hline $\mathrm{O} 3-\mathrm{H} 5 \cdots \mathrm{O} 4$ & $0.94(7)$ & $1.65(7)$ & $2.489(6)$ & $147(6)$ \\
$\mathrm{C} 11-\mathrm{H} 11 \cdots \mathrm{O} 2$ & 0.98 & 2.12 & $2.793(7)$ & 125 \\
$\mathrm{C} 11-\mathrm{H} 11 \cdots \mathrm{O} 2^{\mathrm{i}}$ & 0.98 & 2.51 & $3.362(8)$ & 146 \\
$\mathrm{C} 2-\mathrm{H} 2 \cdots \mathrm{O} 2^{\mathrm{ii}}$ & 0.93 & 2.62 & $3.458(8)$ & 151 \\
\hline
\end{tabular}

Symmetry codes: (i) $-x+1,-y+1,-z$; (ii) $x-1, y, z$.

Table 2

Details of $\pi-\pi$ interactions: intercentroid distances $(\AA)$.

$C g 1$ and $C g 2$ are centroids of the $\mathrm{C} 1-\mathrm{C} 6$ and $\mathrm{O} 1 / \mathrm{C} 5-\mathrm{C} 9$ rings, respectively.

$\begin{array}{lll}C g 1 \cdots C g 2^{\mathrm{i}} & 3.498(7)\end{array}$

$C g 1 \cdots C g 2^{\mathrm{ii}} \quad 3.539(7)$

This trend had previously been observed in the similar structure of 2-difluoroacetyl-1,3-cyclohexadione (Grieco et al., 2011), in which the difluoration reaction affects the $\mathrm{C} 10-\mathrm{C} 11$ distance [1.529 (2) $\AA$ ] .

\section{Supramolecular features}

In the crystal structure of (I), the molecules are assembled in a head-to-tail overlapping manner as a result of the $\pi-\pi$ interactions between the benzene and lactone rings of neighbouring molecules (Table 2) into stacks along the $b$-axis direction (Fig. 2). The observed stacking arrangement can be considered as a balance between van der Waals dispersion and repulsion interactions, and electrostatic interactions between two rings of opposed polarity - the benzene ring (high electron density) and the lactone ring (low electron density)

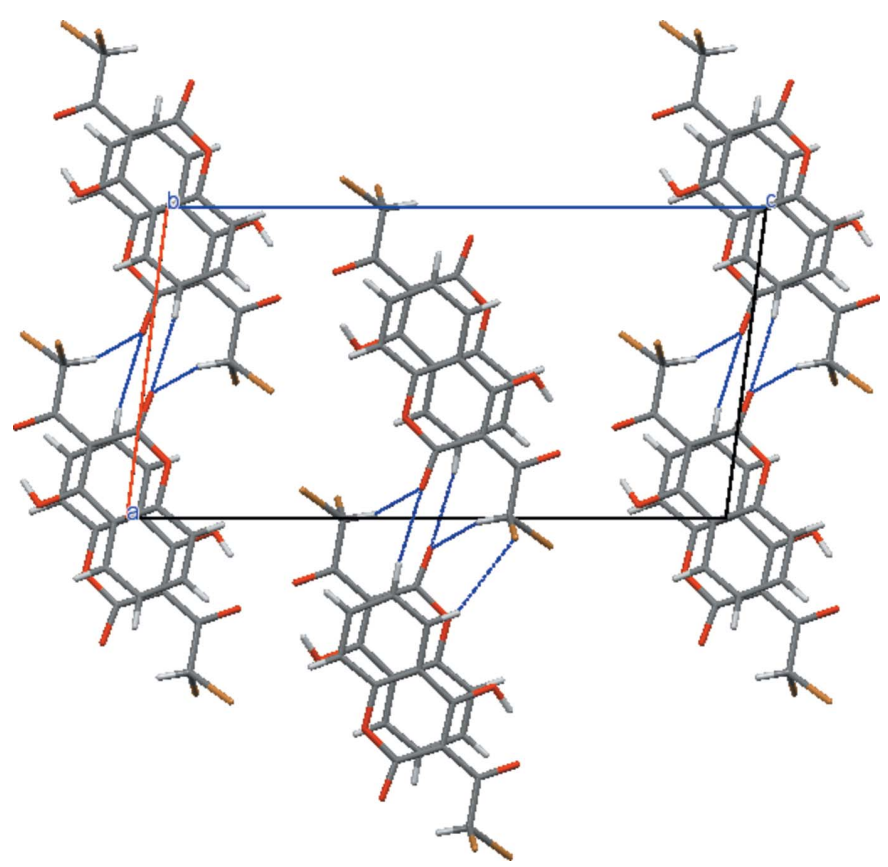

Figure 3

The crystal packing, viewed down the $b$ axis, showing the intermolecular $\mathrm{C}-\mathrm{H} \cdots \mathrm{O}$ hydrogen bonds as thin blue lines. 
<smiles>CC(=O)c1c(O)c2ccccc2oc1=O</smiles>

Figure 4

The synthetic route for (I).

(Hunter \& Sanders, 1990). Weak intermolecular C-H...O hydrogen bonds (Table 2) further link these stacks into layers parallel to the $a b$ plane (Fig. 3).

\section{Synthesis and crystallization}

An excess amount of bromine dissolved in acetic acid was added dropwise to a solution of 3-acetyl-4-hydroxy- $2 \mathrm{H}$ chromen-2-one in acetic acid (Fig. 4). During the reaction, the dropwise addition was made after every disappearance of the brown colour of the bromine. The reaction mixture was maintained under stirring at $373 \mathrm{~K}$ until the bromine colour persisted. The resulting solution was left to crystallize at room temperature to obtain transparent crystals of a light-yellow colour. Yield: $70 \%$; m.p. $=375 \mathrm{~K}$.

\section{Refinement}

Crystal data, data collection and structure refinement details are summarized in Table 3. The hydroxy atom H5 was located from an electron density difference map and freely refined. Cbound $\mathrm{H}$ atoms were fixed geometrically $(\mathrm{C}-\mathrm{H}=0.93$ or $0.98 \AA$ ) and refined as riding, with $U_{\text {iso }}(\mathrm{H})$ set to $1.2 U_{\text {eq }}$ of the parent atom.

\section{Acknowledgements}

Professor A. Ben Salah is acknowledged for his contribution to the X-ray diffraction data collection at the Laboratory of Materials Science and the Environment, University of Sfax, Tunisia.

\section{References}

Bruker (2001). SMART and SAINT. Bruker AXS Inc., Madison, Wisconsin, USA.

Cravotto, G., Nano, G. M., Palmisano, G. \& Tagliapietra, S. (2001). Tetrahedron Asymmetry, 12, 707-709.

Dimmock, J. R., Vashishtha, S. C. \& Stables, J. P. (2000). Eur. J. Med. Chem. 35, 241-248.
Table 3

Experimental details.

\begin{tabular}{|c|c|}
\hline \multicolumn{2}{|l|}{ Crystal data } \\
\hline Chemical formula & $\mathrm{C}_{11} \mathrm{H}_{6} \mathrm{Br}_{2} \mathrm{O}_{4}$ \\
\hline$M_{\mathrm{r}}$ & 361.98 \\
\hline Crystal system, space group & Monoclinic, $P 2_{1} / n$ \\
\hline Temperature $(\mathrm{K})$ & 296 \\
\hline$a, b, c(\AA)$ & $9.399(4), 6.916(3), 17.967(7)$ \\
\hline$\beta\left({ }^{\circ}\right)$ & $97.37(3)$ \\
\hline$V\left(\AA^{3}\right)$ & $1158.4(8)$ \\
\hline$Z$ & 4 \\
\hline Radiation type & Мо $K \alpha$ \\
\hline$\mu\left(\mathrm{mm}^{-1}\right)$ & 7.00 \\
\hline Crystal size $(\mathrm{mm})$ & $0.15 \times 0.12 \times 0.10$ \\
\hline \multicolumn{2}{|l|}{ Data collection } \\
\hline Diffractometer & $\begin{array}{l}\text { Bruker SMART CCD area } \\
\text { detector }\end{array}$ \\
\hline Absorption correction & $\begin{array}{l}\text { For a sphere (Win } G X \text {; Farrugia, } \\
\text { 2012) }\end{array}$ \\
\hline$T_{\min }, T_{\max }$ & $0.58,0.75$ \\
\hline $\begin{array}{l}\text { No. of measured, independent and } \\
\text { observed }[I>2 \sigma(I)] \text { reflections }\end{array}$ & $11685,3234,1094$ \\
\hline$R_{\text {int }}$ & 0.089 \\
\hline$(\sin \theta / \lambda)_{\max }\left(\AA^{-1}\right)$ & 0.712 \\
\hline \multicolumn{2}{|l|}{ Refinement } \\
\hline$R\left[F^{2}>2 \sigma\left(F^{2}\right)\right], w R\left(F^{2}\right), S$ & $0.057,0.133,0.96$ \\
\hline No. of reflections & 3234 \\
\hline No. of parameters & 158 \\
\hline $\mathrm{H}$-atom treatment & $\begin{array}{l}\mathrm{H} \text { atoms treated by a mixture of } \\
\text { independent and constrained } \\
\text { refinement }\end{array}$ \\
\hline$\Delta \rho_{\max }, \Delta \rho_{\min }\left(\mathrm{e} \AA^{-3}\right)$ & $0.43,-0.48$ \\
\hline
\end{tabular}

Computer programs: SMART and SAINT (Bruker, 2001), SHELXS97 and SHELXL97 (Sheldrick, 2008), ORTEP-3 for Windows and WinGX (Farrugia, 2012).

Dou, H. J. M., Vernin, G. \& Metzger, J. (1969). J. Heterocycl. Chem. 6, 575-576.

Farrugia, L. J. (2012). J. Appl. Cryst. 45, 849-854.

Grieco, L. M., Halliday, G. A., Junk, C. P., Lustig, S. R., Marshall, W. J. \& Petrov, V. A. (2011). J. Fluor. Chem. 132, 1198-1206.

Hunter, C. A. \& Sanders, J. K. M. (1990). J. Am. Chem. Soc. 112, 5525-5534.

Kayser, O. \& Kolodziej, H. (1997). Planta Med. 63, 508-510.

Lyssenko, K. A. \& Antipin, M. Yu. (2001). Russ. Chem. Bull. 50, 418431.

Ploypradith, P., Mahidol, C., Sahakitpichan, P., Wongbundit, S. \& Ruchirawat, S. (2004). Angew. Chem. Int. Ed. 43, 866-868.

Sheldrick, G. M. (2008). Acta Cryst. A64, 112-122.

Takase, K., Sasaki, K., Shimizu, K. \& Nozoe, T. (1971). Bull. Chem. Soc. Jpn, 44, 2460-2464.

Traven, V. F., Manaev, A. V., Safronova, O. B., Chibisova, T. A., Lyssenko, K. A. \& Antipin, M. Yu. (2000). Russ. J. Gen. Chem. 70, 798-808.

Wang, C. J., Hsieh, Y. J., Chu, C. Y., Lin, Y. L. \& Tseng, T. H. (2002). Cancer Lett. 183, 163-168. 


\section{supporting information}

Acta Cryst. (2015). E71, 121-123 [doi:10.1107/S2056989014027947]

\section{Crystal structure of 3-(2,2-dibromoacetyl)-4-hydroxy-2H-chromen-2-one}

\section{Ameni Brahmia, Afef Ghouili and Rached Ben Hassen}

\section{Computing details}

Data collection: SMART (Bruker, 2001); cell refinement: SMART (Bruker, 2001); data reduction: SAINT (Bruker, 2001); program(s) used to solve structure: SHELXS97 (Sheldrick, 2008); program(s) used to refine structure: SHELXL97 (Sheldrick, 2008); molecular graphics: ORTEP-3 for Windows (Farrugia, 2012); software used to prepare material for publication: WinGX (Farrugia, 2012).

\section{3-(2,2-Dibromoacetyl)-4-hydroxy-2H-chromen-2-one}

\section{Crystal data}

$\mathrm{C}_{11} \mathrm{H}_{6} \mathrm{Br}_{2} \mathrm{O}_{4}$

$M_{r}=361.98$

Monoclinic, $P 2_{1} / n$

Hall symbol: -P 2 yn

$a=9.399$ (4) $\AA$

$b=6.916(3) \AA$

$c=17.967(7) \AA$

$\beta=97.37(3)^{\circ}$

$V=1158.4(8) \AA^{3}$

Data collection

Bruker SMART CCD area-detector diffractometer

Radiation source: fine-focus sealed tube

Graphite monochromator

$\varphi$ and $\omega$ scans

Absorption correction: for a sphere

(WinGX; Farrugia, 2012)

$T_{\min }=0.58, T_{\max }=0.75$

Refinement

Refinement on $F^{2}$

Least-squares matrix: full

$R\left[F^{2}>2 \sigma\left(F^{2}\right)\right]=0.057$

$w R\left(F^{2}\right)=0.133$

$S=0.96$

3234 reflections

158 parameters

0 restraints

Primary atom site location: structure-invariant

direct methods
$Z=4$

$F(000)=696$

$D_{\mathrm{x}}=2.076 \mathrm{Mg} \mathrm{m}^{-3}$

Melting point: $375 \mathrm{~K}$

Mo $K \alpha$ radiation, $\lambda=0.71073 \AA$

$\mu=7.00 \mathrm{~mm}^{-1}$

$T=296 \mathrm{~K}$

Needle, yellow

$0.15 \times 0.12 \times 0.10 \mathrm{~mm}$

11685 measured reflections

3234 independent reflections

1094 reflections with $I>2 \sigma(I)$

$R_{\text {int }}=0.089$

$\theta_{\max }=30.4^{\circ}, \theta_{\min }=2.3^{\circ}$

$h=-10 \rightarrow 13$

$k=-6 \rightarrow 8$

$l=-25 \rightarrow 25$

Secondary atom site location: difference Fourier map

Hydrogen site location: inferred from neighbouring sites

$\mathrm{H}$ atoms treated by a mixture of independent and constrained refinement

$w=1 /\left[\sigma^{2}\left(F_{\mathrm{o}}^{2}\right)+(0.0409 P)^{2}+0.259 P\right]$ where $P=\left(F_{\mathrm{o}}{ }^{2}+2 F_{\mathrm{c}}{ }^{2}\right) / 3$

$(\Delta / \sigma)_{\max }<0.001$

$\Delta \rho_{\max }=0.43$ e $\AA^{-3}$

$\Delta \rho_{\min }=-0.48$ e $\AA^{-3}$ 


\section{Special details}

Geometry. All e.s.d.'s (except the e.s.d. in the dihedral angle between two 1.s. planes) are estimated using the full covariance matrix. The cell e.s.d.'s are taken into account individually in the estimation of e.s.d.'s in distances, angles and torsion angles; correlations between e.s.d.'s in cell parameters are only used when they are defined by crystal symmetry. An approximate (isotropic) treatment of cell e.s.d.'s is used for estimating e.s.d.'s involving 1.s. planes.

Refinement. Refinement of $F^{2}$ against ALL reflections. The weighted $R$-factor $w R$ and goodness of fit $S$ are based on $F^{2}$, conventional $R$-factors $R$ are based on $F$, with $F$ set to zero for negative $F^{2}$. The threshold expression of $F^{2}>\sigma\left(F^{2}\right)$ is used only for calculating $R$-factors (gt) etc. and is not relevant to the choice of reflections for refinement. $R$-factors based on $F^{2}$ are statistically about twice as large as those based on $F$, and $R$ - factors based on ALL data will be even larger.

Fractional atomic coordinates and isotropic or equivalent isotropic displacement parameters $\left(\hat{A}^{2}\right)$

\begin{tabular}{lllll}
\hline & $x$ & $y$ & $z$ & $U_{\text {iso }} * / U_{\text {eq }}$ \\
\hline Br1 & $0.56249(8)$ & $0.89000(12)$ & $0.15244(5)$ & $0.0906(3)$ \\
Br2 & $0.58984(10)$ & $0.45607(13)$ & $0.21230(4)$ & $0.1029(4)$ \\
O1 & $0.1772(4)$ & $0.7341(5)$ & $-0.05777(19)$ & $0.0525(10)$ \\
O2 & $0.4010(5)$ & $0.6891(6)$ & $-0.0136(2)$ & $0.0637(12)$ \\
O3 & $0.0411(5)$ & $0.6852(6)$ & $0.1484(2)$ & $0.0639(12)$ \\
O4 & $0.2923(5)$ & $0.6171(7)$ & $0.2042(2)$ & $0.0782(14)$ \\
C1 & $-0.1597(7)$ & $0.7718(8)$ & $0.0249(3)$ & $0.0552(16)$ \\
H1 & -0.1941 & 0.7656 & 0.0711 & $0.066^{*}$ \\
C2 & $-0.2515(7)$ & $0.8099(8)$ & $-0.0390(4)$ & $0.0609(17)$ \\
H2 & -0.3490 & 0.8267 & -0.0365 & $0.073^{*}$ \\
C3 & $-0.1981(8)$ & $0.8233(8)$ & $-0.1075(3)$ & $0.0590(17)$ \\
H3 & -0.2612 & 0.8507 & -0.1504 & $0.071^{*}$ \\
C4 & $-0.0559(7)$ & $0.7973(8)$ & $-0.1138(3)$ & $0.0533(16)$ \\
H4 & -0.0219 & 0.8061 & -0.1601 & $0.064^{*}$ \\
C5 & $0.0358(7)$ & $0.7576(7)$ & $-0.0489(3)$ & $0.0453(15)$ \\
C6 & $-0.0123(6)$ & $0.7421(7)$ & $0.0202(3)$ & $0.0426(14)$ \\
C7 & $0.0922(7)$ & $0.7009(8)$ & $0.0839(3)$ & $0.0476(15)$ \\
C8 & $0.2351(7)$ & $0.6807(8)$ & $0.0762(3)$ & $0.0448(14)$ \\
C9 & $0.2804(8)$ & $0.7008(8)$ & $0.0022(3)$ & $0.0489(15)$ \\
C10 & $0.3352(7)$ & $0.6382(8)$ & $0.1427(3)$ & $0.0576(17)$ \\
C11 & $0.4958(7)$ & $0.6271(9)$ & $0.1383(3)$ & $0.0637(18)$ \\
H11 & 0.5117 & 0.5825 & 0.0883 & $0.076^{*}$ \\
H5 & $0.117(8)$ & $0.628(9)$ & $0.180(3)$ & $0.08(2)^{*}$ \\
& & & & \\
\hline
\end{tabular}

Atomic displacement parameters $\left(\AA^{2}\right)$

\begin{tabular}{lllllll}
\hline & $U^{11}$ & $U^{22}$ & $U^{33}$ & $U^{12}$ & $U^{13}$ & $U^{23}$ \\
\hline $\mathrm{Br} 1$ & $0.0551(5)$ & $0.1001(6)$ & $0.1139(7)$ & $-0.0050(4)$ & $0.0000(4)$ & $-0.0223(5)$ \\
$\mathrm{Br} 2$ & $0.1112(8)$ & $0.1317(8)$ & $0.0614(5)$ & $0.0584(6)$ & $-0.0057(4)$ & $0.0091(4)$ \\
$\mathrm{O} 1$ & $0.044(3)$ & $0.071(3)$ & $0.042(2)$ & $0.005(2)$ & $0.004(2)$ & $0.0054(18)$ \\
$\mathrm{O} 2$ & $0.044(3)$ & $0.093(3)$ & $0.055(3)$ & $0.010(2)$ & $0.008(2)$ & $0.006(2)$ \\
$\mathrm{O} 3$ & $0.061(3)$ & $0.088(3)$ & $0.044(3)$ & $0.006(3)$ & $0.012(2)$ & $-0.003(2)$ \\
O4 & $0.066(3)$ & $0.130(4)$ & $0.037(2)$ & $0.005(3)$ & $0.003(2)$ & $0.001(2)$ \\
C1 & $0.052(5)$ & $0.054(4)$ & $0.062(4)$ & $-0.001(3)$ & $0.017(4)$ & $-0.007(3)$ \\
C2 & $0.048(4)$ & $0.054(4)$ & $0.078(5)$ & $0.003(3)$ & $-0.005(4)$ & $-0.002(3)$ \\
C3 & $0.062(5)$ & $0.053(4)$ & $0.058(4)$ & $0.002(3)$ & $-0.006(4)$ & $0.006(3)$
\end{tabular}


supporting information

\begin{tabular}{lllllll} 
C4 & $0.046(5)$ & $0.054(4)$ & $0.058(4)$ & $0.000(3)$ & $0.001(3)$ & $0.004(3)$ \\
C5 & $0.045(4)$ & $0.035(4)$ & $0.055(4)$ & $0.001(3)$ & $0.003(3)$ & $0.004(3)$ \\
C6 & $0.048(4)$ & $0.036(4)$ & $0.043(4)$ & $-0.003(3)$ & $0.006(3)$ & $-0.003(2)$ \\
C7 & $0.059(5)$ & $0.046(4)$ & $0.040(4)$ & $-0.008(3)$ & $0.016(3)$ & $-0.004(3)$ \\
C8 & $0.050(4)$ & $0.054(4)$ & $0.031(3)$ & $0.001(3)$ & $0.007(3)$ & $0.000(3)$ \\
C9 & $0.054(5)$ & $0.045(4)$ & $0.048(4)$ & $0.005(3)$ & $0.007(4)$ & $0.000(3)$ \\
C10 & $0.062(5)$ & $0.064(4)$ & $0.045(4)$ & $0.005(3)$ & $0.002(4)$ & $-0.001(3)$ \\
C11 & $0.058(5)$ & $0.086(5)$ & $0.044(4)$ & $0.011(4)$ & $-0.006(3)$ & $-0.004(3)$ \\
\hline
\end{tabular}

Geometric parameters $\left(\AA,{ }^{\circ}\right)$

\begin{tabular}{|c|c|c|c|}
\hline $\mathrm{Br} 1-\mathrm{C} 11$ & $1.930(6)$ & $\mathrm{C} 2-\mathrm{H} 2$ & 0.9300 \\
\hline $\mathrm{Br} 2-\mathrm{C} 11$ & $1.910(6)$ & $\mathrm{C} 3-\mathrm{C} 4$ & $1.368(8)$ \\
\hline $\mathrm{O} 1-\mathrm{C} 5$ & $1.368(6)$ & $\mathrm{C} 3-\mathrm{H} 3$ & 0.9300 \\
\hline $\mathrm{O} 1-\mathrm{C} 9$ & $1.374(6)$ & $\mathrm{C} 4-\mathrm{C} 5$ & $1.386(7)$ \\
\hline $\mathrm{O} 2-\mathrm{C} 9$ & $1.206(7)$ & $\mathrm{C} 4-\mathrm{H} 4$ & 0.9300 \\
\hline $\mathrm{O} 3-\mathrm{C} 7$ & $1.315(6)$ & $\mathrm{C} 5-\mathrm{C} 6$ & $1.379(7)$ \\
\hline $\mathrm{O} 3-\mathrm{H} 5$ & $0.94(7)$ & $\mathrm{C} 6-\mathrm{C} 7$ & $1.438(7)$ \\
\hline $\mathrm{O} 4-\mathrm{C} 10$ & $1.233(7)$ & $\mathrm{C} 7-\mathrm{C} 8$ & $1.375(8)$ \\
\hline $\mathrm{C} 1-\mathrm{C} 2$ & $1.370(7)$ & $\mathrm{C} 8-\mathrm{C} 10$ & $1.453(7)$ \\
\hline $\mathrm{C} 1-\mathrm{C} 6$ & $1.413(8)$ & $\mathrm{C} 8-\mathrm{C} 9$ & $1.454(8)$ \\
\hline $\mathrm{C} 1-\mathrm{H} 1$ & 0.9300 & $\mathrm{C} 10-\mathrm{C} 11$ & $1.523(9)$ \\
\hline $\mathrm{C} 2-\mathrm{C} 3$ & $1.391(8)$ & $\mathrm{C} 11-\mathrm{H} 11$ & 0.9800 \\
\hline $\mathrm{C} 5-\mathrm{O} 1-\mathrm{C} 9$ & $121.8(5)$ & $\mathrm{C} 1-\mathrm{C} 6-\mathrm{C} 7$ & $123.8(5)$ \\
\hline $\mathrm{C} 7-\mathrm{O} 3-\mathrm{H} 5$ & $103(4)$ & $\mathrm{O} 3-\mathrm{C} 7-\mathrm{C} 8$ & $123.5(5)$ \\
\hline $\mathrm{C} 2-\mathrm{C} 1-\mathrm{C} 6$ & $119.7(6)$ & $\mathrm{O} 3-\mathrm{C} 7-\mathrm{C} 6$ & $115.4(6)$ \\
\hline $\mathrm{C} 2-\mathrm{C} 1-\mathrm{H} 1$ & 120.2 & $\mathrm{C} 8-\mathrm{C} 7-\mathrm{C} 6$ & $121.1(5)$ \\
\hline $\mathrm{C} 6-\mathrm{C} 1-\mathrm{H} 1$ & 120.2 & $\mathrm{C} 7-\mathrm{C} 8-\mathrm{C} 10$ & $118.4(5)$ \\
\hline $\mathrm{C} 1-\mathrm{C} 2-\mathrm{C} 3$ & $119.6(6)$ & $\mathrm{C} 7-\mathrm{C} 8-\mathrm{C} 9$ & $119.1(5)$ \\
\hline $\mathrm{C} 1-\mathrm{C} 2-\mathrm{H} 2$ & 120.2 & $\mathrm{C} 10-\mathrm{C} 8-\mathrm{C} 9$ & $122.5(6)$ \\
\hline $\mathrm{C} 3-\mathrm{C} 2-\mathrm{H} 2$ & 120.2 & $\mathrm{O} 2-\mathrm{C} 9-\mathrm{O} 1$ & $114.7(5)$ \\
\hline $\mathrm{C} 4-\mathrm{C} 3-\mathrm{C} 2$ & $122.2(6)$ & $\mathrm{O} 2-\mathrm{C} 9-\mathrm{C} 8$ & $127.1(6)$ \\
\hline $\mathrm{C} 4-\mathrm{C} 3-\mathrm{H} 3$ & 118.9 & $\mathrm{O} 1-\mathrm{C} 9-\mathrm{C} 8$ & $118.2(6)$ \\
\hline $\mathrm{C} 2-\mathrm{C} 3-\mathrm{H} 3$ & 118.9 & $\mathrm{O} 4-\mathrm{C} 10-\mathrm{C} 8$ & $120.7(6)$ \\
\hline $\mathrm{C} 3-\mathrm{C} 4-\mathrm{C} 5$ & $117.7(6)$ & $\mathrm{O} 4-\mathrm{C} 10-\mathrm{C} 11$ & $118.7(5)$ \\
\hline $\mathrm{C} 3-\mathrm{C} 4-\mathrm{H} 4$ & 121.1 & $\mathrm{C} 8-\mathrm{C} 10-\mathrm{C} 11$ & $120.6(6)$ \\
\hline $\mathrm{C} 5-\mathrm{C} 4-\mathrm{H} 4$ & 121.1 & $\mathrm{C} 10-\mathrm{C} 11-\mathrm{Br} 2$ & $111.6(4)$ \\
\hline $\mathrm{O} 1-\mathrm{C} 5-\mathrm{C} 6$ & $122.1(5)$ & $\mathrm{C} 10-\mathrm{C} 11-\mathrm{Br} 1$ & $104.7(4)$ \\
\hline $\mathrm{O} 1-\mathrm{C} 5-\mathrm{C} 4$ & $115.7(5)$ & $\mathrm{Br} 2-\mathrm{C} 11-\mathrm{Br} 1$ & $112.2(3)$ \\
\hline $\mathrm{C} 6-\mathrm{C} 5-\mathrm{C} 4$ & $122.2(6)$ & $\mathrm{C} 10-\mathrm{C} 11-\mathrm{H} 11$ & 109.4 \\
\hline $\mathrm{C} 5-\mathrm{C} 6-\mathrm{C} 1$ & $118.7(5)$ & $\mathrm{Br} 2-\mathrm{C} 11-\mathrm{H} 11$ & 109.4 \\
\hline $\mathrm{C} 5-\mathrm{C} 6-\mathrm{C} 7$ & $117.5(6)$ & $\mathrm{Br} 1-\mathrm{C} 11-\mathrm{H} 11$ & 109.4 \\
\hline
\end{tabular}

Hydrogen-bond geometry $\left(\AA,{ }^{\circ}\right)$

\begin{tabular}{lllll}
\hline$D-\mathrm{H} \cdots A$ & $D-\mathrm{H}$ & $\mathrm{H} \cdots A$ & $D \cdots A$ & $D-\mathrm{H} \cdots A$ \\
\hline $\mathrm{O} 3-\mathrm{H} 5 \cdots \mathrm{O} 4$ & $0.94(7)$ & $1.65(7)$ & $2.489(6)$ & $147(6)$
\end{tabular}


supporting information

\begin{tabular}{lllll}
$\mathrm{C} 11-\mathrm{H} 11 \cdots \mathrm{O} 2$ & 0.98 & 2.12 & $2.793(7)$ & 125 \\
$\mathrm{C} 11-\mathrm{H} 11 \cdots \mathrm{O} 2^{\mathrm{i}}$ & 0.98 & 2.51 & $3.362(8)$ & 146 \\
$\mathrm{C} 2-\mathrm{H} 2 \cdots \mathrm{O} 2^{\mathrm{ii}}$ & 0.93 & 2.62 & $3.458(8)$ & 151 \\
\hline
\end{tabular}

Symmetry codes: (i) $-x+1,-y+1,-z$; (ii) $x-1, y, z$. 